\title{
Publicaciones españolas para la fundamentación de un marco teórico sobre los estudios de usuarios de información
}

\author{
Isabel Villaseñor Rodríguez *
}

Artículo recibido: 19 de agosto de 2013. Artículo aceptado: 7 de febrero de 2014.

\section{Resumen}

En el presente trabajo se identifican y analizan los documentos publicados en España desde el año 1993, en forma de monografía, ponencia o artículo, que pueden arrojar luz sobre la teoría de los estudios de usuarios de información entendidos como la actividad que se desarrolla con el fin de conocer las necesidades, usos y grado de satisfacción de los usuarios de información. Se describen las fuentes más utilizadas y se analiza el tipo de acercamiento al tema, si general o específico. Se estudian, también, rasgos característicos de la autoría de esos trabajos así como el desarrollo cronológico de los mismos.

* Universidad Complutense de Madrid, España. isavilla@ucm.es 
Palabras clave: Estudios de Usuarios de Información; Teoría de los Estudios de Usuarios de Información.

\section{Abstract}

Spanish publications providing grounding and theoretical framework for research on information users Isabel Villaseñor-Rodríguez

Monographs, papers and articles published in Spain since 1993 are examined to shed theoretical light on the approach to information users as a function of meeting user needs and measuring the degree of user satisfaction. The paper describes the most commonly cited sources and discusses the approach to the topic, whether general or specific. Characteristic features of authorship of these writings as well as their chronological development are also examined.

Keywords: Studies of Users of Information; Studies of Users of Information Theory.

\section{INTRODUCCIÓN}

T os estudios de usuarios de información se enmarcan en lo que Calva ${ }^{1}$ ha Ldado en llamar "el fenómeno de las necesidades de información". Se trata de una actividad que, mediante la aplicación de uno o varios métodos (con sus correspondientes técnicas y herramientas), busca el conocimiento (identificación y caracterización) de todo lo relativo a las necesidades informativas del ser humano; esto es: el surgimiento de la necesidad de información, la manifestación de la necesidad a través del comportamiento del usuario en la búsqueda de esa información y la satisfacción o insatisfacción de la necesidad. Es una actividad, pues, que aporta nuevo conocimiento utilizando un método científico para ello, ambos rasgos definitorios de una investigación. Delgado López-Cózar afirma que son trabajos de investigación "aquellos que 
utilizando una metodología más o menos precisa describen los métodos científicos empleados y presentan resultados originales que aportan conocimiento". ${ }^{2}$

La experiencia en el ámbito de la Biblioteconomía y la Documentación respecto a este asunto se ubica, esencialmente, en el plano de la práctica profesional. Se trata de investigación de carácter experimental o aplicada, en cuanto que da a la luz trabajos empíricos basados en estudios de campo interesados en la solución de problemas específicos en situaciones reales, que no aportan elementos necesarios para establecer un marco teórico que sirva de referencia. Tales contribuciones no suelen establecer relaciones generales y causales que generen conocimiento teórico aplicable al fenómeno de las necesidades de información y que sea aprovechable por las unidades de información para satisfacer los requerimientos informativos de sus usuarios. De ahí que se pretenda influir en el aspecto teórico de cara a sentar bases sólidas para desarrollos concretos posteriores.

La literatura sobre el tema es controvertida con referencia a la validación y universalidad de los resultados obtenidos, y no expresa mucha consistencia con referencia a la terminología. La inexistencia de una base teórica explica las limitaciones de esta línea de investigación y el que se haya centrado en asuntos tangibles tales como la demanda y el uso de información y no en cuestiones más abstractas como las necesidades de información. En los últimos años (finales del siglo XX) se ha dado un esfuerzo en este sentido con la creación de distintos modelos basados en la investigación empírica, que pretenden proporcionar un marco de referencia para el estudio del usuario de información. Es el caso del modelo NEIN, desarrollado por Juan José Calva González.

A partir del análisis de una base de datos mexicana, Patricia Hernández Salazar concluye que los trabajos sobre estudios de usuarios son o bien descripciones de experiencias específicas o análisis bibliográficos de la obra de autores anglosajones; que la mayoría de los trabajos no precisan conceptos tales como metodología, método, técnica o instrumento de trabajo; y que no existe consistencia en la conceptuación de términos fundamentales como información, usuario, estudios y formación de usuarios. ${ }^{3}$ Esta autora aboga por la necesidad de desarrollar marcos conceptuales en esta disciplina con base en que los estudios de usuarios presentan, entre otras, las deficiencias siguientes:

2 Emilio Delgado López-Cózar, La investigación en Biblioteconomía y Documentación, p. 167.

3 Véase "El fenómeno de los usuarios de la información", en Filiberto Felipe Martínez Arellano y Juan José Calva González (comps.), La investigación bibliotecológica en la era de la información. Memoria del XXI Coloquio de Investigación Bibliotecológica y de la Información. 24-26 de septiembre de 2003, México: UNAM/CUIB, 2004, pp.165-176. 
- Confusión entre la función y los objetivos.

- Falta de conocimiento sobre las técnicas a emplear o empleadas, que no son bien aplicadas.

- No captan los datos que se necesitan.

- Carecen de rigor en aspectos estadísticos tales como la determinación de las muestras y el análisis de los resultados.

Aurora González Teruel habla de las críticas hechas a la investigación en necesidades y usos de la información ya desde la década de $1960 .{ }^{4}$ Entre estas críticas destaca las referidas a la escasa aplicabilidad de sus resultados y el uso de metodologías inadecuadas. La mayoría de los estudios se dirigen a la investigación de la necesidad de un determinado documento o al uso de un determinado recurso informativo en vez de decirnos sobre lo que necesitan los usuarios para solucionar problemas concretos; tampoco nos dicen nada de aquellos usuarios que, aun necesitando información, han preferido optar por otra estrategia o han decidido no buscarla. Por confundir los términos se han querido estudiar las necesidades a partir de las demandas e incluso del uso de la información (el uso es un indicador parcial de la demanda y ésta, a su vez, un indicador parcial de las necesidades). En cuanto a las metodologías inadecuadas, la técnica empleada con más frecuencia para recoger información es el cuestionario, con el que se obtienen datos cuantitativos pero poca información cualitativa.

Juan José Calva González también denuncia la escasa bibliografía sobre los aspectos teóricos del fenómeno de las necesidades de información y asegura que lo que se dan son trabajos sobre la búsqueda de información de determinados grupos de sujetos, las fuentes y recursos que utilizan. ${ }^{5}$ Según el autor, para satisfacer las necesidades de información en el seno de un centro de información primero hay que conocerlas: hay que detectarlas, identificarlas, analizarlas y desarrollar los mecanismos necesarios para satisfacerlas (dentro o fuera del centro). Entre los errores cometidos en los estudios de usuarios de información que denuncia destacan los siguientes: no se hace una selección representativa de los sujetos a estudiar, se aplican a usuarios reales, dejando a los potenciales; no se estudia cómo el usuario obtiene la información ni cómo la usa, y se estudia sobre todo el comportamiento, no las necesidades de información.

De ahí que nos interese conocer qué asuntos se han publicado en España en relación con este tema, sus aplicaciones y utilidades y la metodología a aplicar en su desarrollo, porque consideramos que es de vital importancia la creación

4 Véase Los estudios de necesidades y usos de la información: fundamentos y perspectivas actuales, Gijón: Trea, 2005.

5 Calva González, op. cit., p. 2. 
de un marco teórico que sistematice la información de la materia. Por eso, en el presente trabajo nos hemos planteado como objetivo esencial analizar aquellas publicaciones españolas que se han acercado a un tratamiento teórico en torno a los estudios de usuarios de información o a alguno de los aspectos implicados en ellos. La muestra que se ofrece es sólo una parte de la investigación que está en proceso ${ }^{6}$ y que se completará ampliando el estudio a un mayor número de ejemplos de las fuentes que aquí se presentan y de otras posibles, como tesis doctorales. Lo que nos ha interesado conocer es el tipo de acercamiento que se ha hecho respecto al tema que nos ocupa; esto es, si general, tratando distintos aspectos, o específico, centrándose en alguno en concreto o presentando alguna propuesta metodológica. Por otra parte, hemos querido identificar el tipo de fuente en que con más frecuencia se dan a conocer estos trabajos: si monografía, acta de congreso o revista, así como el año en que se ha publicado mayor número de ellos y datos referidos a la autoría de los mismos, tales como la nacionalidad, la profesión y la forma de trabajar de sus autores, si individual o colectivamente, y todo con la finalidad de determinar el interés que despiertan este tipo de investigaciones porque consideramos necesario e imprescindible que los resultados de una investigación se hagan públicos (pueden ser de utilidad para profesionales de la información y académicos) y porque creemos que, desgraciadamente, esto no es lo habitual.

Se puede afirmar que, al menos en España, no existen trabajos de idénticas características al presente, de ahí que uno de sus objetivos principales sea sentar las bases para su desarrollo y proliferación de los mismos ya que los consideramos esenciales para el desenvolvimiento de líneas de investigación relacionadas con la teoría de los estudios de usuarios de la información.

\section{Metodología empleada}

El tema del presente trabajo se ha abordado utilizando el método de investigación documental, el cual se basa en la búsqueda y análisis de fuentes escritas;

6 Lo que se ofrece son algunos avances de la investigación que estamos llevando a cabo en el marco del proyecto del Instituto de Investigaciones Bibliotecológicas y de la Información denominado "Análisis de las investigaciones sobre el fenómeno de las necesidades de información en España y México: en distintas comunidades sociales y académicas", dirigido por Juan José Calva González. Por otra parte, queremos que este estudio venga a completar el presentado en una publicación anterior ("Los estudios de usuarios publicados en España en el siglo XXI", en III Seminario de Usuarios de la Información. La investigación sobre las necesidades de información de diferentes comunidades, México: UNAM/CUIB 2009, pp. 3-78), con el que nos propusimos conocer no sólo el grado de difusión de los trabajos dedicados a estudiar a los usuarios de información, sino también aspectos relacionados con ellos tales como la autoría, la fuente donde se publican, los objetivos marcados o la metodología empleada en ellos. 
la información recabada sirve para conocer mejor y saber en qué estado se encuentra un aspecto concreto. Se trata, pues, de una revisión bibliográfica llevada a cabo a partir de distintas fuentes elegidas por su interés.

El primer paso ha sido identificar y seleccionar las fuentes escritas y publicadas que servirán para obtener la información que necesitamos. En este caso, se han utilizado monografías, actas de congresos o jornadas de profesionales de la información y revistas especializadas en Biblioteconomía y Documentación por considerar que todas ellas constituyen un canal de comunicación esencial, no sólo para la puesta en común de experiencias profesionales, sino también para la presentación de líneas de investigación, además de ofrecer información de actualidad. Para la selección de la muestra se han utilizado los criterios de prestigio y continuidad así como el que las fuentes se encontraran publicadas en cualquier formato.

Respecto a las monografías, hemos tenido en cuenta las publicadas hasta el momento. En cuanto a las actas de congresos o jornadas, de todos los eventos existentes hemos seleccionado los seis que tienen más resonancia tanto en el ámbito profesional como en el académico por la calidad de sus organizadores y porque representan una variedad que se basa en tres criterios: encuentros de carácter general y ámbito nacional, encuentros de carácter general pero de ámbito autonómico y encuentros referidos a un tipo de centros de información como son las bibliotecas y de ámbito autonómico.

En referencia a las revistas españolas especializadas en Biblioteconomía y Documentación nos hemos centrado en el estudio de 12 que se encuentran incluidas en las principales base de datos nacionales e internacionales de la especialidad.

Por último, en relación con los límites cronológicos marcados para la búsqueda de la información en las fuentes, hemos partido de una fecha concreta que tiene una significación esencial en el asunto que nos ocupa. Se trata de 1993, año en que Elías Sanz publicó un artículo titulado "La realización de estudios de usuarios: una necesidad urgente", ${ }^{7}$ y que supuso la presentación sistemática y consciente de este tema en el ámbito de la Biblioteconomía y la Documentación españolas. La búsqueda de información se ha hecho teniendo en cuenta la fecha mencionada y se cierra en el mes de junio de 2013, convirtiendo el periodo en 21 años de publicación en España.

Una vez identificadas y seleccionadas las fuentes se ha procedido a la localización de los trabajos que reúnen las características mencionadas y que pueden considerarse como trabajos teóricos sobre el tema. Tras la detenida lectura de los mismos y su análisis de contenido se valoraron los datos aportados. 


\section{Presentación De los Recursos utilizados}

Para obtener la información requerida hemos hecho uso de las fuentes consultándolas directamente. A continuación se describen con el fin de tener conocimiento del contexto en que se encuentran las publicaciones identificadas.

\section{Los encuentros de profesionales}

Con este término nos referimos a todo tipo de evento de carácter periódico en el que los miembros de una asociación formada por profesionales de una actividad laboral se reúnen para debatir cuestiones relativas a la misma y previamente fijadas. Nuestro interés se enfoca en las comunicaciones o ponencias presentadas que, a veces, resultan de difícil acceso porque o no se publican o la publicación no se hace por los canales habituales de difusión, limitándose la edición y la entrega a los participantes del encuentro. En algunas ocasiones pueden aparecer como documento independiente, formando parte de una colección o de un número monográfico de una revista. Si se publican como resultado de la actividad suelen llevar el nombre unitario de "Actas". Estas reuniones se pueden dar a nivel local, nacional o internacional y reciben las denominaciones de Jornadas, Encuentro, Mesas Redondas, etc. Su interés es manifiesto para los ámbitos científico y profesional como canal de comunicación, ya que en ellas se dan a conocer las últimas tendencias y logros, sirviendo no sólo para conocer las investigaciones en curso sino también para mantenerse al día respecto a la profesión e intercambiar experiencias y puntos de vista. Se celebran con cierta frecuencia y en distintos ámbitos, lo que dificulta sistematizar su información. En el caso de España existe una publicación ${ }^{8}$ que ha recogido aquellos eventos dirigidos a los profesionales de la Documentación que se celebran con cierta periodicidad. De todos ellos, para este estudio se han seleccionado aquellos que cuentan con mayor representatividad por ser los mejor valorados tanto por los profesionales españoles de la Biblioteconomía y la Documentación como por los académicos. La mayoría de ellos disfrutan de continuidad y todos publican sus actas:

1. Jornadas Españolas de Documentación

2. Jornadas sobre Gestión de la Información y del Conocimiento

3. Jornadas Andaluzas de Documentación

8 C. Tejada Artigas y L. Rodríguez Yunta, "Recursos de Internet sobre desarrollo profesional en Documentación 4. Reuniones científicas, congresos, jornadas, simposios y seminarios en España”, en Revista Española de Documentación Científica, vol. 29 (1), 2006, pp.153-173. 
4. Jornades Catalanes d'Informació i Documentació

5. Jornadas Bibliotecarias de Andalucía

6. Jornadas Bibliotecarias de la Comunidad de Madrid

Salvo las últimas, todas son jornadas organizadas por asociaciones profesionales: FESABID (Federación Española de Sociedades de Archivística, Biblioteconomía, Documentación y Museística), SEDIC (Sociedad Española de Documentación e Información Científica), AAD (Asociación Andaluza de Documentalistas), COBDC (Col-legi Oficial de Bibliotecaris-Documentalistes de Catalunya) y AAB (Asociación Andaluza de Bibliotecarios), dándose el caso de que la primera, FESABID, representa a un amplio y variado conjunto de asociaciones profesionales. Las Jornadas Bibliotecarias de la Comunidad de Madrid son responsabilidad de un organismo público (la Subdirección General de Bibliotecas de la Comunidad de Madrid) y son las únicas que restringen la participación a los profesionales de las bibliotecas dependientes de ese organismo. El resto, aunque algunas lleven en su denominación algún rasgo determinante (ya geográfico, ya de institución informativa), son abiertas en su participación y en su temática. El haber seleccionado las Jornadas Bibliotecarias de la Comunidad de Madrid se debe al importante papel que han jugado en la consolidación del sistema bibliotecario de esa Comunidad Autónoma por establecer criterios comunes consensuados para todas las bibliotecas dependientes de ese sistema bibliotecario (determinante para el resto del país).

Las Jornadas Españolas de Documentación se celebran cada dos años y de ellas hemos analizado desde la IV (1994) hasta la XIII (mayo de 2013).

Las Jornadas sobre Gestión de la Información y del Conocimiento se celebran anualmente. Se han analizado las ponencias publicadas desde su primera edición (1999) hasta la XV (2013).

Las Jornadas Andaluzas de Documentación se han celebrado bajo ese nombre en tres ediciones $(1997,1999,2003)$, para pasar a llamarse desde 2005 Foro de especialistas en Información y Documentación en Andalucía. Con este nuevo nombre se han celebrado anualmente en ocho ocasiones. Para nuestro estudio analizamos las ponencias presentadas a este evento desde 1997 hasta 2012, ya que aún no hay información sobre el último, celebrado en el mes de junio de 2013.

Las Jornades Catalanes d'Informació i Documentació, de celebración bianual, nos ofrecen nueve ediciones en el marco cronológico propuesto: la $5^{\text {a }}$ (1995), la $6^{\text {a }}(1997)$, la $7^{\text {a }}(1999)$, la $8^{\text {a }}(2001)$, la $9^{a}(2004)$, la $10^{a}(2006)$, la $11^{\text {a }}$ (2008), la $12^{a}(2010)$ y la $13^{a}(2012)$. 
Las Jornadas Bibliotecarias de Andalucía se celebran cada uno o dos años. Se han analizado las ediciones desde la VIII, celebrada en 1994, hasta la XVI, en 2011. Las XVII se celebraron en el mes de octubre de 2013.

Por último, las Jornadas Bibliotecarias de la Comunidad de Madrid han publicado tan sólo las actas de la $3^{a}$ (2002) y $4^{a}$ edición (2004). No obstante, hemos tenido acceso a los contenidos (no publicados) de las ediciones $1^{a}$ (1991) y $2^{\mathrm{a}}(2000)$.

\section{Las revistas especializadas}

Para la identificación de las revistas españolas especializadas en Biblioteconomía y Documentación se ha consultado DICE ${ }^{9}$ (Difusión y Calidad Editorial de las Revistas Españolas de Humanidades y Ciencias Sociales y Jurídicas), base de datos fruto de un convenio de colaboración entre el Consejo Superior de Investigaciones Científicas (CSIC) y la Agencia Nacional de Evaluación de la Calidad y Acreditación (ANECA), ${ }^{10}$ y que tiene el objetivo de facilitar el conocimiento y la consulta de algunas de las características editoriales de las revistas españolas de Humanidades y Ciencias Sociales más estrechamente ligadas a la calidad, relativas a aspectos cualitativos como los mecanismos de evaluación de originales para publicar, la apertura de los órganos de gestión y dirección, la presencia de diversas instituciones no vinculadas a la entidad editora entre las contribuciones publicadas, la difusión de las revistas en bases de datos multidisciplinares y especializadas de prestigio internacional o el tipo de presencia en Internet. La consulta de este recurso se ha contrastado con la de RESH (Revistas Españolas de Ciencias Sociales y Humanidades: Valoración integrada e índice de citas), ${ }^{11}$ la cual aporta en especial datos relativos al uso e influencia de las revistas a partir de la elaboración de índices de citas que permiten calcular el impacto de cada una en el entorno disciplinar más próximo. El hecho de que DICE aporte información

9 Véase http://dice.cindoc.csic.es/

10 Entidad que financia su mantenimiento y ha sido creada por el Grupo de Investigación "Evaluación de publicaciones científicas en Ciencias Sociales y Humanas" del Centro de Información y Documentación Científica (CINDOC), CSIC. ANECA utiliza DICE como referencia de calidad de las publicaciones españolas, en sus procesos de evaluación de profesorado.

11 "Aporta los resultados del análisis de las Revistas Españolas de Ciencias Sociales y Humanas desde el punto de vista de su calidad. Presenta en primer lugar los Índices de Citas correspondientes a los años 1999, 2000, 2001, 2002 y 2003 a través de los cuales puede valorarse el uso y la influencia de cada una de las revistas que aparecen citadas. A partir de las revistas citadas, con un simple clic, se puede acceder a los datos bibliográficos básicos de las revistas así como a los niveles de cumplimiento de otros parámetros fundamentales de calidad, tanto editorial como de visibilidad internacional, facilitando así una visión de conjunto sobre los diferentes aspectos de la calidad de cada revista, base de una valoración integrada" (Información obtenida de su página web: http://epuc.cchs.csic.es/resh/\#). 
sobre los criterios Latindex (Sistema Regional de Información en Línea para Revistas Científicas de América Latina, el Caribe, España y Portugal) cumplidos por las revistas que describe nos ha hecho considerar fundamentalmente este recurso para seleccionar las revistas especializadas en Biblioteconomía y Documentación con mejor valoración. Nos centraremos en el estudio de 12 de ellas, las cuales se encuentran incluidas en las principales base de datos nacionales e internacionales de la especialidad. Las revistas seleccionadas, de entre las 39 que analiza DICE y las 33 que recoge RESH, son las siguientes:

1. Anales de Documentación

2. BiD: textos universitaris de biblioteconomia i documentació

3. Boletín de ANABAD

4. Boletín de la Asociación Andaluza de Bibliotecarios

5. Cuadernos de Documentación Multimedia

6. Documentación de las Ciencias de la Información

7. Forinf@. Revista Iberoamericana sobre Usuarios de Información

8. Ítem. Revista de biblioteconomía i documentació

9. El Profesional de la Información

10. Revista Española de Documentación Cientifica

11. Revista General de Información y Documentación

12. Scire: Representación y Organización del Conocimiento

Todas ellas representan tres sectores de la actividad desarrollada en Biblioteconomía y Documentación: el académico, el de la investigación y el profesional. Hemos de decir que la publicación número 7 se ha incluido omitiendo algunos de los criterios establecidos para la selección de las fuentes: se trata de una revista electrónica (no impresa) que está por debajo de otras en cuanto a criterios Latindex cumplidos; sin embargo, hay que tener en cuenta que es la única revista española dedicada exclusivamente a temas relacionados con los usuarios de la información. Todas ellas acogen colaboraciones procedentes tanto del mundo académico como del profesional.

Anales de Documentación es una revista editada por la Facultad de Comunicación y Documentación de la Universidad de Murcia. Su periodicidad ha sido anual hasta el año 2010 y semestral desde 2011. Tiene dos ediciones complementarias: la impresa, que se ha publicado hasta el año 2010, y la electrónica, disponible en la página electrónica de la Universidad de Murcia. ${ }^{12}$ Se trata de una revista especializada en cualquiera de las ramas de las 
Ciencias de la Información Documental y su primer número corresponde a 1998, de forma que, dados los límites cronológicos establecidos, hemos consultado los trabajos publicados durante 16 años, de 1998 a 2013. Esta publicación cumple con 33 criterios Latindex en su versión tradicional y con 36 en su edición electrónica.

La revista BiD: textos universitaris de biblioteconomia $i$ documentació es obra de la Facultat de Biblioteconomia i Documentació de la Universidad de Barcelona. Se publica desde 1998 y es de periodicidad semestral. Cumple con 30 criterios Latindex. Para nuestro estudio hemos tenido en cuenta los números publicados desde 1998 hasta junio de 2013.

El Boletín de ANABAD es una publicación de la Federación Española de Asociaciones de Archiveros, Bibliotecarios, Museólogos y Documentalistas que recoge información relacionada con cada una de las materias asociadas a la actividad de los distintos profesionales que integran la asociación. Es de periodicidad trimestral y se publica desde el año 1950, por lo que se trata de la revista especializada más antigua de España. Para la elaboración de este trabajo hemos podido cubrir el periodo completo de 1993 a enero de 2013. Esta publicación cumple con 19 criterios Latindex.

Por su parte, el Boletín de la Asociación Andaluza de Bibliotecarios es una revista de periodicidad trimestral (y semestral en sus dos últimos años) que, editada por la misma asociación, se centra en artículos, recensiones y noticias relacionadas con el mundo de la Biblioteconomía y la Documentación. El primer número apareció en 1984 y el último en el año 2011. Dados los límites cronológicos hemos analizado los trabajos publicados durante 19 años, de 1993 a 2011. Este boletín cumple 27 criterios Latindex.

Cuadernos de Documentación Multimedia es una publicación de la Universidad Complutense de Madrid que pretende tratar la documentación desde un punto de vista relacionado fundamentalmente con las nuevas tecnologías. Es de periodicidad anual y publicó su primer número en 1992, con el nombre Cuadernos de documentación audiovisual. A partir del número 2, correspondiente a 1993, comenzó a utilizarse la denominación actual. El último número (23) se publicó en 2012. Esta revista cumple con 32 criterios Latindex.

La revista Documentación de las Ciencias de la Información es responsabilidad del Departamento de Biblioteconomía y Documentación de la Facultad de Ciencias de la Información de la Universidad Complutense de Madrid. Se publica desde 1976 y su periodicidad es anual. Cumple con 33 criterios Latindex. Para el presente trabajo hemos analizado 20 volúmenes (desde 1993 a 2012).

En cuanto a Forinf@. Revista Iberoamericana sobre Usuarios de Información cabe decir que es la revista electrónica del Foro Internacional de Trabajo 
con Usuarios (FITCU). Se publica desde 1998 y es responsabilidad del Laboratorio de Estudios Métricos de Información del Departamento de Biblioteconomía y Documentación de la Universidad Carlos III. Su periodicidad es trimestral y no se publica desde 2007. Cumple con 27 criterios Latindex. Además de los artículos se han tenido en cuenta otras secciones, tales como "Editorial" o "Comunicaciones", ya que se trata de la única revista española especializada en el tema de los estudios de usuarios de información.

El Col-legi Oficial de Bibliotecaris-Documentalistes de Catalunya publica Ítem. Revista de biblioteconomía i documentació desde 1987, en ella se recogen publicaciones referentes a todos los campos de actividad de los profesionales asociados. Cumple con 31 criterios Latindex, es de periodicidad cuatrimestral hasta el año 2007 y semestral desde 2008. Para el presente trabajo hemos tenido en cuenta los números publicados de 1993 a enero-junio de 2012, que es el último aparecido.

El Profesional de la Información, de periodicidad bimestral y semestral, versa sobre información, bibliotecas y nuevas tecnologías de la información. Su primer número apareció en 1992 con el título Information World en español (IWE) para cambiar al actual en 1998. Dados los límites cronológicos de nuestra investigación, hemos analizado los trabajos publicados de 1993 a enero-junio de 2013. Esta publicación cumple 33 criterios Latindex y es la única revista de edición privada con la que contamos.

El Instituto de Estudios Documentales sobre Ciencia y Tecnología (IEDCYT) del Consejo Superior de Investigaciones Científicas (CSIC) publica la Revista Española de Documentación Científica (REDC) desde 1977 y su periodicidad es trimestral. Cumple con 35 criterios Latindex. Hemos analizado los artículos publicados desde 1993 hasta junio de 2013.

La Revista General de Información y Documentación es publicada por la Facultad de Ciencias de la Documentación de la Universidad Complutense de Madrid. Se creó en 1992 y su periodicidad ha sido semestral hasta el año 2008, convirtiéndose en anual desde 2009. Recoge los trabajos generados por la investigación propia y ajena a la institución, así como los aspectos de toda índole relacionados con el ámbito académico de la misma. Cumple 32 criterios Latindex. Los volúmenes analizados abarcan desde el año 1993 hasta el año 2013.

Por último, la revista Scire: Representación y Organización del Conocimiento está editada por la Red Ibersid ${ }^{13}$ y se publica desde el año 1995. Es semestral y versa sobre la representación, normalización, tratamiento, recuperación y comunicación de la información y el conocimiento. Hemos analizado los

13 Foro internacional de periodicidad anual, cuyo objetivo es facilitar el encuentro entre profesionales, investigadores, docentes y estudiantes de las Ciencias de la Documentación y de otras disciplinas relacionadas. 
trabajos presentados desde el año de su creación hasta el primer número de 2013. Cuenta con 33 criterios Latindex.

\section{ANÁLISIS DE LAS PUbLICACIONES}

\section{La publicación de monografías}

Es evidente que la monografía ofrece, entre otras ventajas, un desarrollo más profundo del tema que es objeto de estudio y una mayor difusión. Si la monografía se hace además con la finalidad de difundir un asunto poco conocido y en un ámbito como el académico, con la intención de servir de material de apoyo a la formación en el aula, el valor es mayor. En este sentido, sobre el tema que nos ocupa tan sólo contamos en España con tres monografías publicadas por profesores universitarios de la especialidad y de la materia. La primera en aparecer fue Manual de estudios de usuarios, de Elías Sanz Casado; la segunda, con 11 años de diferencia, la obra de Aurora González Teruel titulada Los estudios de necesidades y usos de la información: fundamentos y perspectivas actuales. Esta misma autora, junto con Maite Barrios Cerrejón, publicó en 2012 el manual Métodos y técnicas para la investigación del comportamiento informacional. Fundamentos y nuevos desarrollos. En las dos primeras publicaciones encontramos un acercamiento histórico-conceptual al tema con un marcado carácter introductorio cuya finalidad es servir de apoyo a la docencia, ya que se trata de dos manuales. La última resulta un excelente trabajo de aplicación práctica y comparte con las anteriores su finalidad. Manual de estudios de usuarios supuso el primer trabajo completo aparecido en España sobre los estudios de usuarios de información y es uno de los más citados dentro de nuestro ámbito ${ }^{14}$ se trata de una introducción descriptiva a la historia, al concepto y a la metodología a emplear en los estudios de usuarios de información. La segunda obra completó con detalle los planteamientos expuestos, haciendo hincapié en el uso de metodologías cualitativas aplicables a los estudios de usuarios poco habituales en nuestro país, además de centrar su interés en uno de los componentes de tales estudios: el comportamiento informativo. El texto de González Teruel y Barrios Cerrejón, aunque no entra en disquisiciones conceptuales, se centra en técnicas metodológicas aplicables al estudio de ese mismo elemento.

14 Según Javier Salvador Bruna, es el segundo libro más citado en España en el periodo que va de 1996 a 2006 ("Top ten. Diez años de investigación española en Biblioteconomía y Documentación (1996-2006)", en Revista General de Información y Documentación, núm. 2, 2007, p. 163). 
Es evidente que no existe un criterio uniforme en ellas con respecto al concepto y al término utilizado para referirse a esta línea de investigación de la Biblioteconomía y la Documentación. Lo que sí tienen en común es el marcado interés por describir los métodos y las técnicas adoptados de otras Ciencias Sociales y que son aplicables a los estudios de usuarios de información, quizá por la escasa experiencia en ello. Delgado López Cózar, ${ }^{15}$ preocupado por saber en qué medida la Biblioteconomía y la Documentación poseen naturaleza científica, manifestó que la investigación en estas disciplinas en nuestro país se encuentra en un estado embrionario. A partir del estudio de lo publicado (la literatura científica generada) llega a la conclusión de que los métodos más empleados son la encuesta, el histórico y el teórico, aunque a partir de la década de 1980 empiezan a tener importancia los métodos bibliométricos; estas disciplinas se encuentran dominadas por metodologías puramente descriptivas (el qué y el cómo), orientadas a resolver los problemas de la práctica bibliotecaria (investigación aplicada) y aplicadas por autores que cuentan con una marcada carencia en su formación metodológica.

\section{La publicación de ponencias}

De los seis encuentros analizados en sus distintas ediciones, tan sólo hemos encontrado en cuatro de ellos alguna ponencia de nuestro interés.

La VI edición de las Jornadas Españolas de Documentación, celebrada en 1998 , nos ofrece tres trabajos que tratan temas relacionados con la teoría de los estudios de usuarios de información. Estas ponencias son:

- "Los estudios de usuarios en los programas de gestión de calidad. Propuesta de un marco teórico integrador para el estudio del usuario de información" (1998)

- "El cuestionario estructurado como herramienta básica para la evaluación de las instituciones documentales" (1998)

- "La representación del usuario en la recuperación de la información" (1998)

15 Véanse sus obras "Los métodos de investigación empleados en la literatura científica producida en Biblioteconomía y Documentación", en I Congreso Universitario de Ciencias de la Documentación. Teoría, Historia y Metodología de la Documentación en España (1975-2000), Madrid: Departamento de Biblioteconomía y Documentación, Universidad Complutense de Madrid, 2000, pp.625-641; La investigación en biblioteconomía y documentación, Gijón: Trea, 2002; "La investigación por encuesta en Biblioteconomía y Documentación española: análisis de las encuestas publicadas en revistas y congresos de la especialidad entre 1976 y 1997", en Metodologías de investigación en Información y Documentación, Salamanca: Universidad, 2004. 
Las dos primeras ponencias son obra de los mismos autores, todos ellos relacionados con el mundo universitario y de la investigación, y que han colaborado en otras ocasiones en relación con el tema de los estudios de usuarios de información: Mónica Izquierdo Alonso, Joaquín Ruíz Abellán y José Tomás Piñera Lucas. En la primera ponencia parten de la consideración del usuario como eje central del modelo de gestión de calidad total, considerada desde la perspectiva de la calidad aplicada a la realidad del proceso informativo-documental en todas sus fases (creación, tratamiento, difusión, uso e influencia de la información). Los autores subrayan la importancia de los estudios de usuarios para conseguir con éxito la implantación de un programa de gestión de calidad. Señalan el mundo de los usuarios como un fenómeno multidimensional con la existencia de un número importante de determinantes a valorar a la hora de realizar un estudio de usuarios. Por eso estiman necesario definir un marco teórico interdisciplinar con la integración y complementariedad de enfoques; máxime cuando no existe un marco conceptual claramente reconocible para el estudio sistemático del usuario de información. Los autores proponen un modelo metodológico para el estudio del usuario desde la perspectiva del proceso comunicativo documental basándose en el análisis de los autores, las categorías de documentos, los tipos de usuarios y las relaciones entre estos tres elementos. También destacan la importancia de la metodología cualitativa basada en la percepción del ser humano para estudiar los procesos que determinan la activación de una necesidad, los factores que favorecen que esa necesidad se transforme en demanda, los comportamientos individuales o colectivos de los usuarios ante la información, la mecánica de la creación de hábitos informativos, las características del uso de la información, etcétera.

La segunda ponencia se aleja de esa aproximación teórica y conceptual para presentar una propuesta metodológica muy concreta y referida a la elaboración de cuestionarios. Partiendo de la afirmación de que esta herramienta se utiliza con frecuencia (y deficientemente) como soporte metodológico para evaluar instituciones documentales, los autores nos ofrecen un examen detallado de todas las etapas que intervienen en su elaboración, ofreciéndonos unas directrices para un diseño riguroso y científico.

La tercera ponencia ofrece un estudio muy completo de las corrientes teóricas que más han influido en la visión que se ha tenido del usuario de los sistemas de recuperación de información. Su autora, María José López-Huertas Pérez, parte del convencimiento de que existe la necesidad de incorporar al usuario en el diseño y elaboración de los sistemas de recuperación de la información, y analiza la evolución experimentada en la teoría de la recuperación de la información desde el interés por el objeto al interés por 
el sujeto (usuario). Presenta también una descripción muy completa de sistemas de recuperación de información basados en el usuario (algunos tesauros y clasificaciones).

Por su parte, en relación con las Jornades Catalanes d'Informació $i$ Documentació, de todas las ediciones incluidas en el marco cronológico propuesto, únicamente la $6^{a}$ y la $9^{a}$ ofrecen dos trabajos que podemos considerar:

- "L'auditoria de la información com a eina per millorar l'input d'informació dels usuaris en un entorn corporatiu" ("La auditoría de la información como una herramienta para mejorar el input de la información de los usuarios en un entorno corporativo") (1997)

- "L'auditoria del coneixement com a pas previ per definir un projecte de gestió del coneixement: metodología" ("La auditoría del conocimiento como paso previo para definir un proyecto de gestión del conocimiento: metodología”) (2004)

Las dos ponencias tienen en común el uso de una terminología novedosa y un acercamiento parcial al tema que nos ocupa, en cuanto que afrontan el análisis de una aplicación concreta de los estudios de usuarios de información en el entorno empresarial.

La primera de las ponencias, obra de las documentalistas Núria Casaldàliga Rojas y Cristina Soy i Aumatell, presenta un estudio sobre qué se entiende por auditoría de la información, ${ }^{16}$ para qué sirve, en qué casos debe usarse, estado de la cuestión y análisis de algunos casos prácticos. El trabajo se completa con algunas indicaciones metodológicas para su puesta en práctica y algunas de las herramientas que se usan (entrevistas y cuestionarios).

Por su parte, la segunda ponencia propone una metodología para auditar el conocimiento de una organización con el fin de diseñar un proyecto de gestión del mismo. La autora, Montserrat García Alsina, documentalista de una empresa privada, describe detalladamente los aspectos a tener en cuenta antes, durante y después de la auditoría y reflexiona sobre los beneficios que ésta puede ofrecer así como sobre los riesgos que comporta. Además de ofrecer un modelo de cuestionario para recabar datos, distingue entre auditoría de la información y auditoría del conocimiento ${ }^{17}$ y señala que una y otra tienen en cuenta las necesidades de información de los usuarios.

16 Término que se refiere al proceso/herramienta que permite determinar el uso que hacen las organizaciones de la información, quiénes son los clientes, de qué recursos informativos dispone, cómo se distribuye la información, por qué se utiliza y quiénes la gestionan y controlan, con la finalidad de conseguir establecer una política de información en la empresa.

17 Se ocupa de la información ya tratada y se centra en las personas y en la cultura empresarial que propicia la creación de conocimiento. 
Las Jornadas Bibliotecarias de Andalucía nos ofrecen un ejemplo de los trabajos buscados:

- "Nuevas demandas del usuario" (2002)

La autora, Roser Lozano, bibliotecaria de profesión, ofrece una reflexión en torno al cambio experimentado en la relación usuario-biblioteca pública. Analiza los fenómenos que explican la transformación de la biblioteca pública centrada en la oferta de servicios a la biblioteca pública centrada en la demanda del usuario. Señala el nuevo papel desempeñado por esta institución y subraya la importancia de llevar a cabo estudios de usuarios y de su entorno, fundamentalmente de los no usuarios.

Por último, de todas las ponencias analizadas de las Jornadas Bibliotecarias de la Comunidad de Madrid, destacamos una que tiene que ver con el asunto que nos interesa y que lleva por título:

- "Evaluación y calidad de los servicios" (2002)

Se trata de un trabajo presentado por un equipo de bibliotecarios de bibliotecas públicas. Los autores, convencidos de la necesidad de la calidad basada en orientar los servicios hacia la satisfacción de las expectativas y necesidades de los usuarios, proponen una serie de herramientas que permitirían evaluar la biblioteca en su conjunto a partir de la evaluación de las necesidades de la comunidad. Se ofrecen dos modelos de evaluación (cualitativa y cuantitativa), así como dos tipos de herramientas (registro de datos y cuestionario) descritos con gran detalle. La finalidad de la propuesta es poder normalizar acciones en este sentido y en todas las bibliotecas públicas de esta Comunidad Autónoma.

Según podemos observar, en las distintas ediciones de las jornadas analizadas únicamente encontramos siete trabajos que podrían considerarse como estudios teóricos en torno al fenómeno de las necesidades de información; de entre todos los encuentros, son las Jornadas Españolas de Documentación las que ofrecen un mayor número de registros (tres). Lo podemos ver en la Tabla 1.

Tabla 1

\begin{tabular}{|l|l|c|}
\hline \multicolumn{1}{|c|}{ Jornadas } & \multicolumn{1}{|c|}{ Periodo } & Publicaciones \\
\hline $\begin{array}{l}\text { Jornadas Españolas de Documentación } \\
\text { (bianual) }\end{array}$ & $\begin{array}{l}\text { Desde la IV edición (1994) hasta la XIII } \\
\text { (mayo de 2013) }\end{array}$ & 3 \\
\hline $\begin{array}{l}\text { Jornadas sobre Gestión de la Información y } \\
\text { del Conocimiento (anual) }\end{array}$ & $\begin{array}{l}\text { Desde la I edición (1999) hasta la XV } \\
\text { (2013) }\end{array}$ & 0 \\
\hline
\end{tabular}




\begin{tabular}{|l|l|c|}
\hline $\begin{array}{l}\text { Jornadas Andaluzas de Documentación } \\
\text { (irregular)/ }\end{array}$ & $\begin{array}{l}\text { Desde la I edición (1997) hasta la VIII } \\
\text { Foro de especialistas en Información y } \\
\text { Documentación en Andalucía (anual) }\end{array}$ & 0 \\
\hline $\begin{array}{l}\text { Jornades Catalanes d'Informació i } \\
\text { Documentació (bianual) }\end{array}$ & $\begin{array}{l}\text { Desde la 5a edición (1995) hasta la 13 } \\
(2012)\end{array}$ & 2 \\
\hline $\begin{array}{l}\text { Jornadas Bibliotecarias de Andalucía } \\
\text { (anual/bianual) }\end{array}$ & $\begin{array}{l}\text { Desde la VIII edición (1994) hasta la XVI } \\
(2011)\end{array}$ & 1 \\
\hline $\begin{array}{l}\text { Jornadas Bibliotecarias de la Comunidad de } \\
\text { Madrid (irregular) }\end{array}$ & $\begin{array}{l}\text { Desde la 1ª edición (1991) hasta la 4a } \\
(2004)\end{array}$ & 1 \\
\hline
\end{tabular}

Por otra parte, no parece que exista relación entre el número de estudios publicados y el año en que se celebra cada uno de los eventos (Tabla 2). El primero no aumenta necesariamente con el paso del tiempo, ni siquiera considerando todas las jornadas en su conjunto. Así, encontramos un ejemplo en el 1997, tres en 1998, dos en 2002 y uno en 2004; es decir, son mayoría los años analizados donde no encontramos ejemplos.

Tabla 2

\begin{tabular}{|c|c|}
\hline Año & Publicaciones \\
\hline 1997 & 1 \\
\hline 1998 & 3 \\
\hline 2002 & 2 \\
\hline 2004 & 1 \\
\hline
\end{tabular}

En cuanto al tipo de acercamiento al tema, podemos decir que tan sólo un estudio lo hace de forma generalista mientras que los demás ofrecen el análisis de algún aspecto en concreto (las nuevas demandas del usuario en la biblioteca pública, la contemplación de éste en los sistemas de recuperación de la información o qué se entiende por auditoría de la información) o propuestas metodológicas (cómo se hace un cuestionario, una hoja de registro de datos o una auditoría del conocimiento) (Tabla 3).

Tabla 3

\begin{tabular}{|c|c|}
\hline Acercamiento & Publicaciones \\
\hline Generalista & 1 \\
\hline Específico & 6 \\
\hline
\end{tabular}

Respecto a la autoría (Tabla 4), vemos que todos los autores son españoles y que predominan los profesionales de la información (cuatro trabajos) sobre los docentes universitarios en nuestras materias (tres trabajos). También 
imperan los trabajos hechos en coautoría (cuatro) frente a los redactados individualmente (tres).

Tabla 4

\begin{tabular}{|l|c|}
\hline \multicolumn{1}{|c|}{ Características de los autores } & Publicaciones \\
\hline Nacionalidad española & 7 \\
Otras nacionalidades & 0 \\
\hline Profesionales de la información & 4 \\
Docentes universitarios/investigadores & 3 \\
\hline Trabajo en coautoría & 4 \\
Trabajo individual & 3 \\
\hline
\end{tabular}

\section{La publicación de articulos}

La mayoría de las revistas analizadas nos ofrecen artículos que se refieren al tema de nuestro interés, ya que de las 12 publicaciones seleccionadas tan sólo tres no lo hacen. Estas son: Cuadernos Multimedia, El Profesional de la Información y Scire.

La revista Anales de Documentación nos ofrece los siguientes artículos:

- "El modelado orientado al usuario: una perspectiva global" (1999)

- "La satisfacción del usuario: Un concepto en alza" (2000)

- "El análisis de citas en trabajos de investigadores como método para el estudio del uso de información en bibliotecas” (2001)

El primer trabajo es la traducción de un texto del profesor Tom Wilson, del Departamento de Estudios de la Información de la Universidad de Sheffield (Reino Unido). Se trata de una reflexión sobre el estudio de los usuarios de sistemas de información basados en sistemas informáticos. El autor afirma que el diseño de dichos sistemas constituye algo más que un simple problema técnico o tecnológico. Aunque los fabricantes de equipos informáticos se han interesado en la ergonomía de los equipos informáticos y han diseñado herramientas dotadas con características apropiadas para satisfacer las necesidades psicológicas del ser humano, el autor propone adentrarse en la comprensión de los procesos cognitivos del usuario para desarrollar sistemas más próximos al mismo. De esta manera, su trabajo se centra en el efecto del estilo cognitivo en el comportamiento de la búsqueda de información, lo que revela diferencias entre los comportamientos de personas con diferentes estilos de aprendizaje. El artículo sigue con el análisis del concepto de información para llegar a situar y analizar al usuario de información en diferentes contex- 
tos: social, laboral, familiar, y acaba centrándose en el estudio del usuario en el trabajo.

El segundo artículo es obra de Carina Rey Martín, profesora de la Facultad de Biblioteconomía y Documentación de la Universidad de Barcelona, preocupada por los estudios de usuarios de información como lo demuestran distintas publicaciones sobre este tema. En esta ocasión presenta algunas conclusiones sobre el concepto "satisfacción del usuario" y propone el modelo de satisfacción de Rachel Applegate. Partiendo de la premisa de que los estudios de satisfacción de los usuarios son, en propiedad, estudios de usuarios, admite la eficacia de los mismos como criterios razonables para poder evaluar un sistema de información en un entorno operativo y considera que la forma más adecuada es la encuesta. Al mismo tiempo, admite la falta de un marco conceptual en el ámbito de la Documentación que permita la realización de este tipo de investigaciones, ya que la evidencia demuestra que hay pocos estudios de satisfacción tanto en España como en Europa, y que los que hay no se centran en las bibliotecas sino en las empresas de servicios. Hace referencia a algunos estudios de usuarios centrados en medir la satisfacción, que se han llevado a cabo en algunos países europeos y expone algunas aportaciones doctrinales de distintos autores al concepto de estudios de satisfacción.

Por último, Cristóbal Urbano Salido, profesor de la Facultad de Documentación de la Universidad de Barcelona, nos propone en el tercer artículo el análisis de citas como un método indirecto eficiente para los estudios de usuarios y de necesidades de información, que no interfiere en el comportamiento del colectivo estudiado: los investigadores como usuarios de bibliotecas. Se trata de una revisión bibliográfica y de un acercamiento teórico que ofrece recomendaciones sobre la aplicación de esta técnica.

La revista BiD. Textos universitaris de biblioteconomia $i$ documentació cuenta con dos artículos que ofrecen cierta similitud:

- "Màrqueting i biblioteques: buscant la satisfacció dels usuaris" ("Marqueting y bibliotecas: buscando la satisfacción de los usuarios”) (2009)

- "Les enquestes als usuaris de les biblioteques publiques de Catalunya: comentaris metodològics" ("Las encuestas a los usuarios de las bibliotecas públicas de Cataluña: comentarios metodológicos”) (2009)

Ambos artículos tienen en común su brevedad y que las conclusiones se obtienen a partir de sendas experiencias prácticas. El primero de ellos, obra del profesor universitario Manuel Cuadrado García, recoge unas breves notas sobre el marketing y la necesidad de recabar información de los usuarios. Es cierto que no habla explícitamente de estudios de usuarios, pero concibe 
el marketing como la disciplina orientada a satisfacer los deseos y necesidades de los "consumidores" de información. El segundo de los artículos, obra de Antoni Laporte Roselló, profesional de la información, ofrece una serie de recomendaciones para las buenas prácticas en materia de cuestionarios y otro tipo de "estudios de público" a partir de la presentación de las principales encuestas a usuarios de bibliotecas públicas catalanas hechas en el siglo XXI.

El Boletín de ANABAD nos presenta dos trabajos centrados fundamentalmente en la metodología a emplear en estudios de usuarios de información:

- "Metodología de cuestionarios: principios y aplicaciones" (1993)

- "Integración de cuestionarios en el OPAC: reflexiones sobre su viabilidad" (2004)

En el primero, María Amérigo Cuervo-Arango, del Departamento de Psicología Social de la Universidad Complutense, ofrece los aspectos que considera esenciales a la hora de elaborar un cuestionario, analizando algunas definiciones del concepto. Establece cuatro etapas en la elaboración de esta herramienta al tiempo que se refiere a su presentación, a la forma de plantear las preguntasy a sus tipos, la organización de los temas y la codificación o exposición de datos obtenidos.

Por su parte, Miguel Ángel Sánchez Herrador y Mariano Boza Puerta, asesores de las bibliotecas públicas de Córdoba y Granada respectivamente, presentan en el segundo artículo un estudio teórico en el que se propone la inclusión de cuestionarios de usuarios en un OPAC (catálogo en línea), con el objetivo de incrementar la comunicación entre el usuario y la biblioteca estableciendo un flujo ágil y constante de información que mejore la gestión de la misma. Como recomendaciones, los autores presentan un cuestionario tipo, así como la clase de información que éste proporcionaría y, además, estudian su viabilidad desde distintos puntos de vista: calidad de la información, aspectos relativos a su implementación informática, cuestiones relacionadas con la privacidad de los datos y repercusiones en la toma de decisiones de la biblioteca.

El Boletín de la Asociación Andaluza de Bibliotecarios cuenta con un solo artículo de nuestro interés:

- "Los usuarios de la biblioteca universitaria" (1995)

Sonsoles Celestino, directora de la Biblioteca Universitaria de Sevilla, tras constatar los cambios producidos en el entorno que afecta a las bibliotecas reflexiona sobre lo que realmente esperan los usuarios de ellas, sobre lo que 
puede considerarse como una buena biblioteca y sobre si realmente es posible una biblioteca que responda a las expectativas de usuarios y bibliotecarios. Ofrece un concepto de usuario, reflexiona sobre las necesidades de información de la comunidad universitaria en su conjunto y expone los principios básicos sobre los que, en su opinión, debe asentarse una biblioteca universitaria, entre los que obviamente se encuentran los estudios de usuarios.

La revista Documentación de las Ciencias de la Información nos ofrece tres artículos distanciados en su fecha de publicación:

- “Aportaciones en torno a los usuarios en Documentación" (1998)

- "La evaluación de colecciones: métodos y modelos" (2002)

- “La importancia de la satisfacción del usuarios" (2011)

El primero es obra de los mismos autores de dos ponencias presentadas en las VI Jornadas Españolas de Documentación: Mónica Izquierdo Alonso, Joaquín Ruíz Abellán y José Tomás Piñera Lucas. En esta ocasión ofrecen un extenso trabajo, dividido en dos partes, ${ }^{18}$ que viene a recoger lo dicho en las ponencias mencionadas y que se refiere a los estudios de usuarios de información en general y a la técnica de la encuesta en particular.

El segundo artículo, obra de la profesora universitaria Ana Pérez López, resulta ser un extenso trabajo sobre los distintos métodos y modelos empleados para la evaluación de las colecciones. Entre ellos se encuentran "los centrados en los usuarios", referidos fundamentalmente al uso que hace el usuario de la colección de un centro de información. Habla de técnicas cuantitativas (registro de préstamos) y cualitativas (entrevista, encuesta, observación) con todo detalle.

El tercer y último artículo es obra de Patricia Hernández Salazar, investigadora del Instituto de Investigaciones Bibliotecológicas y de la Información de la Universidad Nacional Autónoma de México. La autora se centra en el estudio conceptual de uno de los elementos de lo que Calva ${ }^{19}$ ha llamado "el fenómeno de las necesidades de información”: la satisfacción del usuario; subraya la necesidad de su estudio y describe posibles métodos y técnicas para ello.

Forinf@. Revista Iberoamericana sobre Usuarios de Información es la publicación que, evidentemente, ofrece más trabajos relacionados con el tema que nos ocupa. Éstos son:

- "Los métodos cualitativos en los estudios de usuarios: una revisión bi-

18 En el encabezado de las páginas interiores del artículo aparece como título del mismo "Dos aportaciones en torno a los usuarios en Documentación”.

19 Véase Calva González, op. cit. 
bliográfica" (1998)

- "Propuesta metodológica en los estudios de usuarios y su aplicación al caso de la industria farmacéutica" (1998)

- "Usuarios y necesidades de información" (2000)

- "Sistema de factores de la conducta del usuario" (2000)

- "Las investigaciones cuantitativas y cualitativas en Ciencia de la Información: algunas consideraciones" (2001)

- "El usuario de la información: unidad de observación mensurable y convergente en los Estudios Métricos de la Información" (2001)

- "Los estudios de usuarios: asignatura pendiente para la Archivística" (2002)

- "El usuario de la información y la biblioteca escolar" (2003)

- "El diseño centrado en el usuario para la creación de productos y servicios de información digital" (2003/2004)

- "Estudios de usuarios y archivística: una alianza lógica" (2004)

- "Perspectiva sistémica de los estudios de usuarios de información" (2005)

El primer artículo es obra de la profesora de Costa Rica Saray Córdoba. En él nos ofrece la enumeración de algunas aportaciones bibliográficas sobre métodos y técnicas no convencionales de aplicación a los estudios de usuarios de información.

El segundo trabajo, escrito por la profesora universitaria María José Ordóñez Vergara, da cuenta del desarrollo de un estudio de usuarios de información. Se centra en la descripción y razonamiento detallados del proceso seguido por la autora en la investigación llevada a cabo con una comunidad de usuarios específica. ${ }^{20}$

El tercero no es propiamente un artículo, sino la traducción de una conferencia impartida por el profesor hindú H. N. Prasad. Se trata de una amplia exposición conceptual acerca del fenómeno de las necesidades de información: surgimiento de la necesidad, comportamiento informativo, uso y satisfacción, etc. El conferencista destaca que este asunto, como disciplina, está poco desarrollado y que se necesita seguir trabajando en su marco conceptual.

El artículo cuarto, obra del investigador cubano Enrique González Suárez, nos ofrece un posible modelo metodológico para afrontar el estu-

20 Los resultados obtenidos los dio a conocer en dos artículos: "La entrevista personal: método para el estudio de usuarios de información”, en Forinf@ Revista Iberoamericana sobre Usuarios de Información, núm. 14, octubre-diciembre, 2001, pp. 6-23 y "El consumo de información en la industria farmacéutica. II. Resultados de una encuesta", en Revista Española de Documentación Científica, vol. 23 (2), 2000, pp.179-196. 
dio de los usuarios de información que denomina "sistema de factores de la conducta del usuario" y que incluye el "entorno científico productivo e informativo del usuario en el entorno social", necesidades de información, "concienciación de las necesidades de información", "motivación informativa", "resolución para ejecutar la actividad informativa", "disposición informativa" y "actividad informativa del usuario, en que se realiza la disposición informativa mediante acciones y operaciones informativas". El autor describe las características de cada uno de los conceptos.

En cuanto al quinto trabajo, coincide con el tercero en el hecho de que se trata del texto de una conferencia. Es obra del profesor cubano Ramadés Linares Columbié. En él se ofrece una breve reflexión sobre el uso de la metodología de investigación cualitativa en lo que llama "Ciencia de la Información”, esto es, la investigación centrada en el usuario como otra posibilidad.

En el sexto trabajo, el investigador mexicano Salvador Gorbea nos ofrece una aproximación a los estudios métricos de la información (Bibliotecometría, Bibliometría, Infometría y Archivometría), mostrando distintas posibilidades de estudio del usuario desde distintos planos.

Respecto al séptimo, obra de la profesora M. Paz Martín Pozuelo, nos encontramos con un editorial donde se reivindica la necesidad de los estudios de usuarios para la Archivística teórica y práctica. Aunque no profundiza en ningún tema, resulta de interés que se contemple este asunto en el ámbito de la Archivística pues, al menos en España, existen muy pocas experiencias tanto en el plano conceptual como en el práctico.

El profesor e investigador mexicano Juan José Calva González nos ofrece, en el octavo estudio, una reflexión muy completa sobre un tipo de usuarios de información poco conocido como es el infantil y adolescente. Plantea algunos asuntos relacionados con su entorno y las investigaciones que se pueden llevar a cabo respecto a este sector.

El noveno artículo, obra del profesor Jesús Tramullas Saz, estudia los conceptos básicos del diseño de sistemas e interfaces para aplicaciones informáticas centrado en el usuario, los distintos enfoques abordados y las técnicas empleadas. Destaca la importancia que tiene el usuario para desarrollar este diseño.

El penúltimo artículo retoma un tema tratado anteriormente en esta revista: la necesidad de considerar los estudios de usuarios en un ámbito en el que no es frecuente encontrarlos, como es el de la Archivística. El profesor y archivero Alfonso Ruiz Cagigal ofrece un pequeño ensayo en el que pretende fundamentar la necesidad de los estudios de usuarios de información para los archivos españoles, donde se dan pocas experiencias en este terreno. Enumera algunas de las aplicaciones que pueden darse y que son de gran valor, como la evaluación del centro, la identificación de grupos de usuarios y 
producción de contenidos, entre otras.

Por último, la documentalista Rita Santaella nos ofrece un artículo donde presenta los estudios de usuarios como subsistema del sistema documental. Señala la necesidad de sistematizar el proceso de un estudio de usuarios para que pueda extrapolarse en investigaciones de similares características. Propone una teoría, basada en un modelo común, que recoge un conjunto de reglas de actuación para operar independientemente del ejemplo concreto al que se aplique que, en su caso, es el de tres archivos de una ciudad española.

La revista Ítem cuenta con dos artículos de interés:

- "Els serveis als usuaris en les biblioteques universitàries" ("Los servicios a los usuarios en las bibliotecas universitarias") (2009)

- "Els usuaris: algunes reflexions al seu voltant" ("Los usuarios: algunas aportaciones en torno a ellos") (2009)

En el primero, las bibliotecarias Cristina Güells y Judit Casals afrontan el estudio de los servicios que deben prestar las bibliotecas universitarias actuales a sus usuarios, teniendo en cuenta fundamentalmente sus necesidades de información y los cambios que ha experimentado su contexto externo. Ofrecen una clasificación muy interesante de estos servicios, según las necesidades y tipos de usuarios.

La profesora Carina Rey Martín parte, en el segundo artículo, de que no existe consenso no sólo en torno a la definición de "usuario" sino también a su tipología, y revisa lo dicho por distintos autores al respecto. Analiza los términos empleados y otros que no han tenido éxito ("consumidor", "cliente"). Tras un breve repaso histórico por su práctica insiste en la necesidad de hacer estudios de usuarios. El artículo se enmarca en un número de la revista que dedica su contenido a los usuarios de información por celebrar la publicación de su $50^{\circ}$ volumen.

La Revista Española de Documentación Científica sólo cuenta con un artículo en todos los volúmenes analizados:

- "La investigación cualitativa y sus aplicaciones en Biblioteconomía y Documentación” (1999)

A partir del estudio y análisis de la temática y técnicas empleadas en los artículos de investigación de dos revistas norteamericanas y una británica, el profesor universitario Ángel Borrego Huerta concluye que la mayor parte de los que emplean técnicas cualitativas estudian el comportamiento del usuario en la recuperación de la información. A partir de ahí expone las 
principales características de las investigaciones que utilizan la metodología cualitativa. Es cierto que no habla expresamente de estudios de usuarios de información, pero sí de una metodología de investigación empleada en ellos.

En cuanto a la Revista General de Información y Documentación podemos decir que se trata de una de las revistas que más artículos recoge relacionados con el tema que nos ocupa. Éstos son:

- "La realización de estudios de usuarios: una necesidad urgente" (1993)

- "Técnicas bibliométricas aplicadas a los estudios de usuarios" (1997)

- "Por qué requerimos una metodología para el estudio de las necesidades de formación e información en las organizaciones y comunidades" (2001)

- "Los estudios de usuarios en los planes de estudio de Biblioteconomía y Documentación” (2007)

- "Metodología de investigación en estudios de usuarios" (2007)

- "Análisis de la Investigación Cualitativa en el área de Biblioteconomía y Documentación (1981-2010)” (2013)

El primer artículo es el que hemos considerado como referente para demarcar cronológicamente nuestra búsqueda ya que puede considerarse como el primero publicado en España que aborda de una manera teórica estos temas. Tras una breve descripción de la evolución de los estudios de usuarios considera qué se entiende por este término y da cuenta de distintas metodologías a aplicar.

En el segundo artículo, Elías Sanz Casado y Carmen Martín Moreno, ambos profesores universitarios, hablan del interés que tiene el uso de técnicas bibliométricas aplicadas al consumo y a la producción científica para la realización de estudios de usuarios de información. A lo largo del trabajo se define qué se entiende por Bibliometría, se hace historia de la aplicación de estas técnicas a los estudios de usuarios y se habla de tipos y aplicaciones de indicadores bibliométricos, en particular a partir de los documentos publicados.

En cuanto al tercer artículo, se trata de un extenso trabajo del profesor cubano Israel A. Núñez Paula, fruto de sus investigaciones bibliográficas sobre tendencias de investigación en el campo de las Ciencias de la Información. A partir de los resultados obtenidos constata dos asuntos en los que centra su interés: por una parte el requerimiento constante de realizar estudios de usuarios, de sus necesidades y expectativas de formación e información para diseñar y desarrollar nuevos servicios y productos; por otra parte, y contradictoriamente, el pobre tratamiento conceptual, metodológico y práctico que se le ha dado a los estudios de usuarios de información.

Los artículos cuarto y quinto son obra de dos profesoras universitarias, 
presentados como ponencias en las XVI Jornadas EUBD/FADOC: Los estudios de usuarios de información. ${ }^{21}$ En el primero de ellos María Luisa Lascurain Sánchez ofrece un brevísimo acercamiento a las asignaturas referidas a los estudios de usuarios de información que se encuentran en los planes de estudio de las titulaciones en Biblioteconomía y Documentación en el curso académico 2006-2007. El resto del artículo se dedica a presentar un breve recorrido histórico de los estudios de usuarios y a exponer lo que, en consideración de la autora, deben ser sus objetivos y contenidos en los planes de estudio. Dedica un apartado a analizar brevemente el papel de este tema en las propuestas de Grado en el marco del Espacio Europeo de Educación Superior. En cuanto al segundo de los artículos, obra de la profesora Carmen Martín Moreno, se trata de un breve compendio teórico sobre los estudios de usuarios que sigue muy de cerca el esquema del libro de Elías Sanz que hemos tomado como referencia: breve recorrido histórico, definición, aplicaciones, métodos y técnicas de recolección de datos... Dedica un brevísimo apartado final a la metodología científica en general y a su aplicación a los estudios de usuarios de información.

El último artículo, escrito por Beatriz Morena de Diago, doctora en Ciencias de la Documentación por la Universidad Complutense de Madrid, es una revisión bibliográfica de algunos trabajos que tratan sobre metodología cualitativa. Aunque no trata específicamente de la teoría de los estudios de usuarios de información, describe algunas técnicas de aplicación a este tipo de investigaciones.

Según vemos en la Tabla 5 , en los distintos volúmenes de las revistas analizadas son 31 los trabajos que podemos considerar para nuestro estudio; de entre todas ellas, es Forinf@ la que ofrece un mayor número de textos (11).

\section{Tabla 5}

\begin{tabular}{|l|c|c|}
\hline \multicolumn{1}{|c|}{ Publicación } & Periodo & Artículos encontrados \\
\hline $\begin{array}{l}\text { Anales de Documentación (anual hasta 2010, semestral } \\
\text { desde 2011). 33 y 36 criterios }\end{array}$ & $1998-2013$ & 3 \\
\hline $\begin{array}{l}\text { BiD. Textos universitaris de biblioteconomia i } \\
\text { documentació (semestral). 30 criterios }\end{array}$ & $1998-2013$ & 2 \\
\hline Boletín de ANABAD (trimestral).19 criterios & $1993-2013$ & 2 \\
\hline $\begin{array}{l}\text { Boletín de la Asociación Andaluza de Bibliotecarios } \\
\text { (trimestral hasta 2009, semestral desde 2010). 27 } \\
\text { criterios }\end{array}$ & $1993-2011$ & 1 \\
\hline
\end{tabular}

21 XVI Jornadas EUBD/FADOC: Los estudios de usuarios de información (15 y 16 de marzo de 2007. Facultad de Ciencias de la Documentación. UCM). Cabe destacar que la sesión académica de las mismas se celebró conjuntamente con el IV Seminario hispano-mexicano de investigación en Bibliotecología y Documentación. 


\begin{tabular}{|l|c|c|}
\hline $\begin{array}{l}\text { Cuadernos de Documentación Multimedia (anual). 32 } \\
\text { criterios }\end{array}$ & $1993-2012$ & 0 \\
\hline $\begin{array}{l}\text { Documentación de las Ciencias de la Información (anual). } \\
33 \text { criterios }\end{array}$ & $1993-2012$ & 3 \\
\hline $\begin{array}{l}\text { Forinf@. Revista Iberoamericana sobre Usuarios de } \\
\text { Información (trimestral). 27 criterios }\end{array}$ & $1998-2007$ & 11 \\
\hline $\begin{array}{l}\text { Item. Revista de biblioteconomía i documentació (cuatri- } \\
\text { mestral hasta 2007, semestral desde 2008). 31 criterios }\end{array}$ & $1993-2012$ \\
\hline $\begin{array}{l}\text { El Profesional de la Información (bimestral y semestral). } \\
\text { 33 criterios }\end{array}$ & $1993-2013$ & 0 \\
\hline $\begin{array}{l}\text { Revista Española de Documentación Científica (trimes- } \\
\text { tral). 35 criterios }\end{array}$ & $1993-2013$ & 1 \\
\hline $\begin{array}{l}\text { Revista General de Información y Documentación (se- } \\
\text { mestral hasta 2008, anual desde 2009). 32 criterios }\end{array}$ & $1993-2013$ & 6 \\
\hline $\begin{array}{l}\text { Scire: Representación y Organización del Conocimiento } \\
\text { (semestral). 33 criterios }\end{array}$ & $1995-2013$ & 0 \\
\hline
\end{tabular}

Por otra parte, no parece que exista relación entre el número de trabajos publicados y el año en que se publican los distintos volúmenes analizados. El primero no aumenta necesariamente con el paso del tiempo, ni siquiera considerando todas las revistas en su conjunto. Sí es cierto que se da la publicación en más variedad de años que en el caso de las jornadas, de forma que, de los 21 años analizados tan sólo en seis $(1994,1996,2006,2008,2010,2012)$ no se encuentran ejemplos. Los años en que más se publicaron artículos relacionados con el tema han sido 2001 y 2009, con cuatro artículos; en segundo lugar están los años 1998, 2000 y 2004, con tres artículos; después, 1993, 1999, 2002 y 2007 con dos y, el resto, con uno (Tabla 6).

Tabla 6

\begin{tabular}{|c|c|c|c|c|c|}
\hline Año & Publicaciones & Año & Publicaciones & Año & Publicaciones \\
\hline 1993 & 2 & 2000 & 3 & 2007 & 2 \\
\hline 1994 & 0 & 2001 & 4 & 2008 & 0 \\
\hline 1995 & 1 & 2002 & 2 & 2009 & 4 \\
\hline 1996 & 0 & 2003 & 1 & 2010 & 0 \\
\hline 1997 & 1 & 2004 & 3 & 2011 & 1 \\
\hline 1998 & 3 & 2005 & 1 & 2012 & 0 \\
\hline 1999 & 2 & 2006 & 0 & 2013 & 1 \\
\hline
\end{tabular}

En cuanto al tipo de acercamiento al tema, podríamos decir que hay 14 trabajos en los que se da de forma general, abordando asuntos tales como 
ámbitos o qué se estudia en las asignaturas dedicadas a la formación de futuros profesionales de la información; y 17 de forma específica, entre los que tenemos ocho que hablan de metodología de recolección de datos (fundamentalmente la cualitativa), dos de tipos de usuarios, tres de técnicas y herramientas de recolección de datos y cuatro de aplicaciones de los estudios de usuarios, entre ellas la evaluación de la colección, el marketing, el diseño centrado en el usuario, el estudio de la satisfacción o la Archivística (Tabla 7).

Tabla 7
\begin{tabular}{|c|c|}
\hline Tipo de acercamiento & Publicaciones \\
\hline Acercamiento generalista & 14 \\
\hline Acercamiento específico & 17 \\
\hline
\end{tabular}

Respecto a la autoría, advertimos mayor variedad en cuanto a la nacionalidad de los autores, dándose el caso de existir nueve trabajos de nacionalidades diferentes a la española (uno de nacionalidad inglesa, otro de nacionalidad hindú, tres de nacionalidad cubana, uno de nacionalidad costarricense y tres de nacionalidad mexicana). La mayor parte de los autores corresponde a la categoría de docentes universitarios/investigadores en Biblioteconomía y Documentación, dándose tan sólo cinco trabajos firmados por profesionales de la información y uno por un doctorando. Esta proporción se observa también en la forma de trabajar, ya que la mayor parte de los trabajos (27) están firmados por un solo autor y cuatro de ellos por más de un autor, de forma que el trabajo individual predomina sobre el trabajo en grupo (Tabla 8)

Tabla 8

\begin{tabular}{|l|c|}
\hline \multicolumn{1}{|c|}{ Parámetros } & Publicaciones \\
\hline Nacionalidad española & 22 \\
Otras nacionalidades & 9 \\
\hline Profesionales de la información & 5 \\
Docentes universitarios/investigadores & 25 \\
Doctorandos & 1 \\
\hline Trabajo en coautoría & 27 \\
Trabajo individual & 4 \\
\hline
\end{tabular}

Por último, dos trabajos son traducciones de conferencias impartidas por sus autores pero se han considerado en el cómputo al haber sido publicados como artículos en revistas españolas, lo que manifiesta el interés de sus responsables por dar a conocer estos temas. 


\section{CONClusiones}

Tras el estudio realizado podemos llegar a las conclusiones siguientes:

1. La publicación de trabajos en España sobre aspectos relacionados con la teoría de los estudios de usuarios de información es escasísima, ya que en los 21 años analizados tan sólo hemos encontrado tres monografías, siete ponencias y 31 artículos.

2. Las monografías publicadas en el periodo seleccionado son de carácter introductorio y responden a un interés didáctico, como apoyo al desarrollo de la docencia de sus autores en la formación universitaria de futuros profesionales de la información.

3. La fuente de carácter periódico más utilizada para dar a conocer trabajos de estas características es la revista, mediante el artículo. Tan sólo siete de los estudios identificados se han dado a conocer en jornadas mientras que 31 ha sido en revistas especializadas. Es cierto que las primeras se celebran con menos frecuencia que la periodicidad en que se publican los distintos volúmenes de las revistas analizadas, pero resulta significativo el hecho de que hayamos encontrado ejemplos de lo buscado en nueve de las 12 revistas y sólo en cuatro de las seis jornadas. Esto nos hace pensar que el artículo es el vehículo más apropiado para afrontar el acercamiento teórico a un tema.

4. El mayor número de ponencias forma parte de las Jornadas Españolas de Documentación, encuentro que goza de más difusión en España ya que está organizado por FESABID (Federación Española de Sociedades de Archivística, Biblioteconomía, Documentación y Museística). Sin embargo, el mayor número de artículos no corresponde a la revista que reúne más criterios Latindex y que se supone es la de mayor prestigio (la Revista Española de Documentación Científica), sino en una de las que cuenta con menos (Forinf@). La razón estriba en que ésta es la única revista española especializada en el tema de los estudios de usuarios de información.

5. En cuanto al año de publicación, podemos decir que pasan cuatro años desde que aparece el artículo de Elías Sanz que hemos tomado como referencia cronológica inicial y tres desde la publicación de la primera monografía sobre el asunto (en 1997 y del mismo autor), hasta que aparecen los primeros trabajos identificados, tanto en jornadas como en revistas. Desde esa fecha (1993) hasta nuestros días tenemos ejemplos de trabajos, exceptuando los años 1996, 2006, 2008 y 2010. Se advierte que el paso de los años no implica un aumento de 
publicaciones en cualquiera de las fuentes estudiadas ya que el mayor número de trabajos corresponde a 1998 (seis); ${ }^{22}$ también es cierto que no se han dejado de publicar, incluso en el último año. Esto puede hacernos pensar que el tema sigue resultando de interés en nuestro ámbito.

6. Respecto al tipo de acercamiento al tema que nos ocupa, predomina el específico, con 24 trabajos (una monografía, seis ponencias y 17 artículos) sobre los 41 identificados. De entre todos los aspectos tratados predomina el análisis de metodologías y técnicas de recolección de datos (una monografía, tres ponencias y ocho artículos). Los demás asuntos tratados son, por orden de interés, el usuario, distintas aplicaciones de los estudios de usuarios y propuestas metodológicas. El resto afronta el tema de forma generalista: dos monografías, una ponencia y 14 artículos.

7. En cuanto a la autoría de los trabajos analizados cabe decir que predominan los autores de nacionalidad española (32), docentes/investigadores universitarios de Biblioteconomía y Documentación (32), que trabajan individualmente (32). De cualquier forma, si consideramos los datos obtenidos teniendo en cuenta la fuente en la que aparecen, vemos que existen diferencias entre una y otra ya que, en el caso de las ponencias, predominan los autores que son profesionales de la información (cuatro de siete), mientras que en los artículos son los autores docentes/investigadores universitarios (26 de 31). Lo mismo ocurre con las monografías, cuyos autores son profesores universitarios. También existen diferencias respecto a la forma de trabajar, ya que en el caso de las ponencias es más frecuente la coautoría (cuatro de tres) mientras que en el caso de los artículos lo es el trabajo individual (27 de 31). En el caso de las tres monografías sólo una está escrita por dos autores. Esto hace pensar que el acercamiento teórico a un tema resulta ser una actividad de pensamiento individual.

8. Se puede afirmar que el marco teórico de los estudios de usuarios de información en España se sitúa fundamentalmente en el ámbito universitario, no sólo porque la mayor parte de los autores de los trabajos identificados sean docentes universitarios/investigadores sino también porque las fuentes que más trabajos han publicado sobre el tema son dos revistas responsabilidad de un departamento

22 Si consideramos que el artículo publicado en la revista Documentación de las Ciencias de la Información en ese año recoge lo mismo que los autores presentaron en dos ponencias durante las VI Jornadas españolas de Documentación podría decirse que en 1998 sólo tenemos cuatro trabajos de interés, al igual que en el año 2002. 
universitario (Forinf@) y de una facultad (Revista General de Información y Documentación Científica).

\section{Bibliografía}

Amérigo Cuervo-Arango, María, "Metodología de cuestionarios: principios y aplicaciones", en Boletín de ANABAD, núm. 3-4, 1993, pp. 263-272.

Borrego Huerta, Ángel, "La investigación cualitativa y sus aplicaciones en Biblioteconomía y Documentación", en Revista Española de Documentación Científica, vol. 22, núm. 2, 1999, pp. 139-156.

Calva González, Juan José, "El usuario de la información y la biblioteca escolar", en Forinf@. Revista Iberoamericana sobre Usuarios de Información, núm. 21, 2003, pp. 6-11.

__ Las necesidades de información. Fundamentos teóricos y métodos, México: UNAM/CUIB, 2004.

Casaldàliga Rojas, Núria; Cristina Soy i Aumatell, "Ĺauditoria de la información com a eina per millorar l'input d'informació dels usuaris en un entorn corporatiu", Ges. Jornades d'Informació i Documentació, Barcelona: Col-legi Oficial de Bibliotecaris-Documentalistes de Catalunya, 1997, pp. 49-63.

Celestino Agudo, Sonsoles, "Los usuarios de la biblioteca universitaria", en Boletín de la Asociación Andaluza de Bibliotecarios, núm. 41, año 11, 1995, pp. 29-38.

Córdoba, Saray, "Los métodos cualitativos en los estudios de usuarios: una revisión bibliográfica", en Forinf@. Revista Iberoamericana sobre Usuarios de Información, núm. 1, julio-septiembre, 1998, pp. 5-8.

Cuadrado García, Manuel, "Màrqueting i biblioteques: buscant la satisfacció dels usuaris", BiD. Textos universitaris de biblioteconomia idocumentació, núm. 23, 2009.

Delgado López-Cózar, Emilio, "Los métodos de investigación empleados en la literatura científica producida en Biblioteconomía y Documentación", en I Congreso universitario de Ciencias de la Documentación. Teoría, Historia y Metodología de la Documentación en España (1975-2000), Madrid: Departamento de Biblioteconomía y Documentación, Universidad Complutense de Madrid, 2000, pp.625-641.

__ La investigación en Biblioteconomía y Documentación, Gijón: Trea, 2002.

"La investigación por encuesta en Biblioteconomía y Documentación española: análisis de las encuestas publicadas en revistas y congresos de la especialidad entre 1976 y 1997", en Metodologías de investigación en Información y Documentación, Salamanca: Ediciones Universidad de Salamanca, 2004. 
García Alsina, Montserrat, "Ĺauditoria del coneixement com a pas previ per definir un projecte de gestió del coneixement: metodología", 9es. Jornades d'Informació i Documentació, Barcelona: Col-legi Oficial de Bibliotecaris-Documentalistes de Catalunya, 2004, pp. 183-206.

García Núñez, María Jesús; Inmaculada Ramón Jiménez et al., "Evaluación y calidad de los servicios", en Terceras Jornadas Bibliotecarias de la Comunidad de Madrid, Madrid, Las Rozas: Consejería de las Artes, Concejalía de Cultura, 2002, pp. 99-148.

González Suárez, Enrique, "Sistema de factores de la conducta del usuario", en Forinf@. Revista Iberoamericana sobre Usuarios de Información, núm. 9, julio-septiembre, 2000, pp. 6-17.

González Teruel, Aurora, Los estudios de necesidades y usos de la información: fundamentos y perspectivas actuales, Gijón: Trea, 2005.

__ ; Maite Barrios Cerrejón, Métodos y técnicas para la investigación del comportamiento informacional. Fundamentos y nuevos desarrollos, Gijón: Trea, 2012.

Gorbea-Portal, Salvador, "El usuario de la información: unidad de observación mensurable y convergente en los Estudios Métricos de la Información", Forinf@. Revista Iberoamericana sobre Usuarios de Información, núm. 13, julio-septiembre, 2001, pp. 4-7.

Güell Guillén, Cristina; Judit Casals Parladé, "Els serveis als usuaris en les biblioteques universitàries”, Ítem, núm. 50, 2009, pp. 67-87.

Hernández Salazar, Patricia, "El fenómeno de los usuarios de la información”, en Filiberto Felipe Martínez Arellano y Juan José Calva González (comps.), La investigación bibliotecológica en la era de la información. Memoria del XXI Coloquio de Investigación Bibliotecológica y de la información. 24-26 de septiembre de 2003, México: UNAM/CUIB, 2004, pp.165-176.

"La importancia de la satisfacción del usuarios", en Documentación de las Ciencias de la Información, vol. 34, 2011, pp. 349-368.

Izquierdo Alonso, Mónica; Joaquín Ruíz Abellán; José Tomás Piñera Lucas, "Los estudios de usuarios en los programas de gestión de calidad. Propuesta de un marco teórico integrador para el estudio del usuario de información", en VI Jornadas Españolas de Documentación. FESABID 98. Los Sistemas de Información al Servicio de la Sociedad, Madrid: FESABID, 1998, pp. 433-444.

__ ; _ ; _, "El cuestionario estructurado como herramienta básica para la evaluación de las instituciones documentales", en VI Jornadas Españolas de Documentación. FESABID 98. Los Sistemas de Información al Servicio de la Sociedad, Madrid: FESABID, 1998, pp. 779-789.

_ _ _ - _ "Aportaciones en torno a los usuarios en Documentación", en Documentación de las Ciencias de la Información, núm. 21, 1998, pp. 11-75. 
Laporte Roselló, Antoni, "Les enquestes als usuaris de les biblioteques públiques de Catalunya: comentaris metodològics”, BiD. Textos universitaris de biblioteconomia i documentació, núm. 23, 2009.

Lascurain Sánchez, María Luisa, "Los estudios de usuarios en los planes de estudio de Biblioteconomía y Documentación”, en Revista General de Información y Documentación, vol. 17, núm. 2, 2007, pp. 151-158.

Linares Columbié, Radamés, "Las investigaciones cuantitativas y cualitativas en Ciencia de la Información: algunas consideraciones", en Forinf@. Revista Iberoamericana sobre Usuarios de Información, núm. 11, enero-marzo, 2001, pp. 11-14.

López-Huertas Pérez, María José, "La representación del usuario en la recuperación de la información", en VI Jornadas españolas de Documentación. FESABID 98. Los Sistemas de Información al Servicio de la Sociedad, Madrid: FESABID, 1998, pp. 521-527.

Lozano, Roser, "Nuevas demandas del usuario", en XII Jornadas Bibliotecarias de Andalucía. Los nuevos retos de los servicios bibliotecarios, Málaga: Asociación Andaluza de Bibliotecarios, 2002, pp. 305-321.

Martín Moreno, Carmen, "Metodología de investigación en estudios de usuarios”, en Revista General de Información y Documentación, vol. 17, núm. 2, 2007, pp. 129-149.

Martín Pozuelo, M. Paz, "Los estudios de usuarios: asignatura pendiente para la Archivística”, en Forinf@. Revista Iberoamericana sobre Usuarios de Información, núm. 17, julio-septiembre, 2002, pp. $4-5$.

Morena de Diago, Beatriz, "Análisis de la Investigación Cualitativa en el área de Biblioteconomía y Documentación (1981-2010)”, en Revista General de Información y Documentación, vol. 23-1, 2013, pp. 43-64.

Núñez Paula, Israel A., "Por qué requerimos una metodología para el estudio de las necesidades de formación e información en las organizaciones y comunidades", en Revista General de Información y Documentación, vol. 11, núm. 1, 2001, pp. 83-108.

Ordóñez Vergara, María José, "Propuesta metodológica en los estudios de usuarios y su aplicación al caso de la industria farmacéutica”, en Forinf@. Revista Iberoamericana sobre Usuarios de Información, núm. 2, octubre-diciembre, 1998, pp. 5-14.

_ - "La entrevista personal: método para el estudio de usuarios de información”, en Forinf@. Revista Iberoamericana sobre Usuarios de Información, núm. 14, octubre-diciembre, 2001, pp.6-23.

_ , "El consumo de información en la industria farmacéutica. II. Resultados de una encuesta”, en Revista Española de Documentación Científica, vol. 23, núm. 2, 2000, pp.179-196.

Prasad, H. N., "Usuarios y necesidades de información”, versión en castellano de Laurie Ann Ortiz Rivera, en Forinf@. Revista Iberoamericana sobre Usuarios de Información, núm. 8, abril-junio, 2000, pp. 12-18. 
Pérez López, Ana, "La evaluación de colecciones: métodos y modelos", en Documentación de las Ciencias de la Información, vol. 25, 2002, pp. 321-360.

Rey Martín, Carina, "Els usuaris: algunes reflexions al seu voltant", Ítem, núm. 50, 2009, pp. 88-101.

__ , "La satisfacción del usuario: Un concepto en alza", en Anales de Documentación, vol. 3, 2000, pp. 138-154.

Ruiz Cagigal, Alfonso, "Estudios de usuarios y archivística: una alianza lógica”, en Forinf@. Revista Iberoamericana sobre Usuarios de Información, núm. 24, 2004, pp. 7-13.

Salvador Bruna, Javier, "Top ten. Diez años de investigación española en Biblioteconomía y Documentación (1996-2006)", en Revista General de Información y Documentación, núm. 2, 2007, pp. 159-182.

Sánchez Herrador, Miguel Ángel; Mariano Boza Puerta, "Integración de cuestionarios en el OPAC: reflexiones sobre su viabilidad", en Boletín de ANABAD, núm. 1-2, 2004, pp. 633-643.

Santaella Ruiz, Rita Dolores, "Perspectiva sistémica de los estudios de usuarios de información”, en Forinf@. Revista Iberoamericana sobre Usuarios de Información, núm. 29, 2005, pp. 7-15.

Sanz Casado, Elías, "La realización de estudios de usuarios: una necesidad urgente", en Revista General de Información y Documentación, vol. 3, núm. 1, 1993, pp.154-166.

__ Manual de estudios de usuarios, Madrid: Fundación Germán Sánchez Ruipérez, Pirámide, 1994.

— ; Carmen Martín Moreno, "Técnicas bibliométricas aplicadas a los estudios de usuarios", en Revista General de Información y Documentación, vol. 7, núm. 2, 1997, pp. 41-68.

Tejada Artigas, C.; L. Rodríguez Yunta, "Recursos de Internet sobre desarrollo profesional en Documentación: 4. Reuniones científicas, congresos, jornadas, simposios y seminarios en España", en Revista Española de Documentación Científica, vol. 29, núm. 1, 2006, pp.153-173.

Tramullas Saz, Jesús, "El diseño centrado en el usuario para la creación de productos y servicios de información digital", en Forinf@. Revista Iberoamericana sobre Usuarios de Información, núm. 22-23, 2003-2004, pp. 6-14.

Urbano Salido, Cristóbal, "El análisis de citas en trabajos de investigadores como método para el estudio del uso de información en bibliotecas", en Anales de Documentación, vol. 4, 2001, pp. 243-266.

Villaseñor Rodríguez, Isabel, "Los estudios de usuarios publicados en España en el siglo XXI", en III Seminario de Usuarios de la Información. La investigación sobre las necesidades de información de diferentes comunidades, México: UNAM/CUIB, 2009, pp. 3-78.

Wilson, Tom, "El modelado orientado al usuario: una perspectiva global", en Anales de Documentación, vol. 2, 1999, pp. 85-94. 